Du bien commun aux biens communs. Approches croisées.

Colloque interdisciplinaire

Université Clermont-Auvergne

5 octobre 2018

\title{
Vers une finance destinée au bien commun : la contribution de l'approche thomiste
}

\author{
Caroline MARIE-JEANNE \\ Maître de Conférences en sciences de gestion \\ Université d'Angers
}

\begin{abstract}
Résumé
L'objet de cet article est d'étudier, à travers la contribution de l'approche thomiste, les liens entre la finance et le bien commun. L'argent n'appartient pas aux biens communs par nature mais il peut être mis en commun par le partage et utilisé en vue du Bien commun au sens philosophique du terme. II s'agit tout d'abord de penser cette notion dans l'ordre financier à partir des écrits fondamentaux. L'étude de l'utilisation de l'argent en vue du Bien commun, selon l'approche thomiste, à travers les prêts et la spéculation, fournit ensuite des pistes de réflexion pour répondre aux défis à venir et définir de nouvelles formes de gestion associées à cette ressource. Saint Thomas met en exergue l'importance du partage de l'argent et du gain, de la gratuité du prêt pour l'existence de la communauté, de la finalité de l'acte et de la valeur d'usage. Nous montrerons enfin comment ces concepts peuvent être développés aujourd'hui pour une finance destinée au Bien commun notamment grâce à la finance participative, à la finance solidaire, au microcrédit, aux social bonds, aux green bonds et aux climate bonds.
\end{abstract}




\section{Vers une finance destinée au bien commun : la contribution de l'approche thomiste}

L'argent n'est pas un bien commun comme les autres, au sens de ressource à partager entre les membres d'une communauté (au pluriel les "biens communs"). Ce concept est d'ailleurs fort différent du "Bien commun » abordé au niveau théologique et philosophique. Il existe ainsi diverses approches du bien commun.

En droit romain, un bien commun est une chose inappropriable par essence comme l'air, ce qui n'est pas le cas de l'argent, typiquement appropriable. Toutefois, pour certains auteurs (Dardot et Laval) aucune chose n'est commune par nature. Une chose est rendue commune par des formes de gestion, des activités de coopération qui nous lient à ces choses et, en même temps, nous lient aussi entre nous. On peut soustraire une chose à la propriété privée aussi bien qu'à la propriété publique pour faire de cette chose un usage qui puisse bénéficier à tous ceux qui sont concernés. Ainsi, dans le cas de la microfinance, l'argent est soustrait au marché pour être réservé à un usage commun. Ces règles de partage de la ressource, présentées dans l'ouvrage Gouvernance des biens communs, font l'objet des travaux d'Elinor Ostrom, Prix Nobel d'économie.

En économie, un bien commun est un bien non exclusif, c'est-à-dire un bien dont on ne peut exclure personne de sa consommation, comme les fonds marins ou l'eau. Nécessaire à tous, il convient d'en offrir l'usage à chacun. Or l'argent n'est a priori ni partagé, ni commun. Par ailleurs, l'économiste américain Paul Samuelson définit le " bien commun " également par le critère de non-rivalité (l'usage par un individu n'empêche pas un même usage ou un autre usage par autrui). II donne l'exemple de l'éclairage public. Or l'argent ne répond pas à ce critère non plus. En effet, la finance est une ressource dont la rivalité est forte (la consommation d'une unité réduit la somme disponible pour les autres), ce qui implique de penser à son renouvellement.

D'ailleurs, l'argent est-il même un « Bien » au sens moral et spirituel du mot ? En soi, l'argent n'est ni bien, ni mal. A la base, il a été créé pour faciliter le troc. Echanger un sac de pommes de terre contre des chaussures semble, en effet, peu commode. Cet instrument d'échange peut alors financer du sens ou du non-sens. La finance semble elle-même neutre; le dictionnaire Larousse la définit comme la " science de la gestion des patrimoines individuels, des patrimoines d'entreprise et des deniers publics". Elle concerne l'ensemble des mécanismes et des institutions qui apportent à l'économie ${ }^{1}$ les capitaux dont elle a besoin pour fonctionner. Le terme finance s'applique à la recherche de financements, à la recherche de placements par les détenteurs de surplus de capitaux et enfin à tous ceux qui organisent la convergence entre ces deux types d'acteurs (marchés financiers, banques...). Son rôle est

\footnotetext{
1 L'économie est définie dans le dictionnaire Larousse comme "l'ensemble des activités d'une collectivité humaine relatives à la production, à la distribution et à la consommation des richesses ».
} 
d'assurer une utilisation optimale des ressources et d'améliorer ainsi le bien-être de chacun. Mais pour les ressources limitées comme l'argent, cette optimisation va se fonder notamment sur le taux de rendement, rapport entre le revenu obtenu et la mise de fonds initiale. Par sa recherche effrénée de la maximisation du gain pécuniaire ${ }^{2}$, au niveau individuel, la finance ne semble parfois plus au service de la communauté. Aussi, peu réputée pour concourir au Bien commun depuis la crise de 2008 , elle est même plutôt citée comme un mal commun et on note une perte de confiance dans les institutions financières. Comment la notion philosophique de Bien commun peut-elle donc se traduire dans une théorie et une pratique financières qui affirment comme finalité de l'action le " bénéfice monétaire " ? En effet, les marchés financiers visent l'accroissement de la richesse matérielle à court terme ${ }^{3}$, alors que la recherche du Bien commun nous procure une croissance humaine et spirituelle à long terme par l'augmentation du bien des autres.

Ainsi, l'argent n'appartient pas par nature aux biens communs (des biens en commun). Mais l'objet de cet article est d'analyser comment il peut contribuer à la réalisation du Bien commun au sens philosophique, notamment en le destinant à un usage commun par l'instauration de règles de partage sur le plan économique. II s'agit ici de redonner à la finance sa véritable place : être un instrument au service de l'économie réelle qui elle-même doit être au service de la communauté.

L'analyse de la notion de bien commun dans l'ordre financier, à la lumière des philosophes, est un préalable nécessaire (I) à l'étude de l'utilisation de l'argent en vue du Bien commun selon l'approche thomiste (II). Les préceptes mis en exergue par le théologien fournissent des pistes de réflexion pour répondre aux défis à venir et définir de nouvelles formes d'utilisation associées à cette ressource (III).

\section{Comment traduire l'idée de bien commun dans l'ordre financier ?}

"La construction de la finance et de la monnaie comme commun est beaucoup plus récente, plus exceptionnelle aussi, que celle de la gestion des ressources dites naturelles, et se situe généralement dans des projets alternatifs", selon Servet. La mise en avant du partage " permet de redonner à la monnaie et à la finance leurs dimensions largement oubliées ou occultées de communs ${ }^{4}$.

Mais pour pouvoir inventer des communs, il convient au préalable de penser le Bien commun par introspection au niveau philosophique à partir des travaux d'Aristote et de Saint Thomas d'Aquin dont on retrouve les préceptes dans la doctrine sociale de l'Eglise.

\footnotetext{
${ }^{2}$ Le gain ou le revenu est au numérateur du ratio dans le calcul du taux de rendement défini par $: \frac{\text { revenu }}{\text { montant investi }}$.

${ }^{3}$ A titre d'exemple, la pratique récente des transactions à haute fréquence consiste à exécuter des ordres boursiers inaccessibles aux analyses humaines en quelques microsecondes grâce à des algorithmes informatiques.

${ }^{4}$ Servet.
} 


\section{A. Le bien commun et l'argent chez Aristote}

Toute la philosophie pratique d'Aristote tend à répondre à sa recherche du bonheur, qui est le bien ultime que poursuit l'homme. Pour lui "le bien pour l'homme consiste dans une activité de l'âme en accord avec la vertu $~^{5}$. Le Bien commun, identifié au juste et à la paix, est l'objectif de toute vraie politique et son respect le critère absolu de la droiture des régimes. Dans l'enseignement d'Aristote le bien suprême est le bien de la communauté.

Aristote $^{6}$ nous amène alors à nous interroger sur le sens de la richesse financière et sur la valeur l'argent. Est-il une fin en soi ou bien seulement un moyen d'échange ? "Cet argent n'est en lui-même qu'une chose absolument vaine, n'ayant de valeur que par la loi et non par la nature, puisqu'un changement de convention parmi ceux qui en font usage peut le déprécier complètement, et le rendre tout à fait incapable de satisfaire aucun de nos besoins. En effet, un homme, malgré tout son argent, ne pourra-t-il pas manquer des objets de première nécessité ? Et n'est-ce pas une plaisante richesse que celle dont l'abondance n'empêche pas de mourir de faim ? C'est comme ce Midas de la mythologie, dont le vœu cupide faisait changer en or tous les mets de sa table » dès qu'il les touchait mais, au milieu de l'abondance, il n'avait plus de quoi apaiser sa faim.

Il distingue alors la chrématistique naturelle qui consiste à acquérir des biens en vue de la satisfaction des besoins et la chrématistique commerciale, plus condamnable, qui consiste à prendre pour finalité l'accumulation illimitée des richesses. De surcroît, il affirme qu'il est contraire à la nature que la monnaie produise de la monnaie. La raison principale de l'interdiction de l'intérêt ${ }^{7}$ est la stérilité de l'argent, l'infécondité du capital. Principe aristotélicien, l'argent, dit-on, n'a pas faculté de s'accroître. Il ne fait pas de petits : Nummus nummum non parit. Il est stérile et $n^{\prime}$ est pas productif. Aristote ${ }^{8}$ est très clair : " on a surtout raison d'exécrer l'usure, parce qu'elle est un mode d'acquisition né de l'argent lui-même, et ne lui donnant pas la destination pour laquelle on l'avait créé. L'argent ne devait servir qu'à l'échange ".

Dans l'optique du bien de la communauté, Aristote prône un système économique vertueux, tout en condamnant les pratiques spéculatives et la recherche effrénée du profit. Toutefois, il s'oppose à Platon pour qui, dans La République, les gardiens de la cité idéale ne possèdent rien en propre et ne sont pas autorisés à acquérir de l'or. Aristote argue, lui, que la communauté des biens génère plus de différends que l'appropriation privée. Mais l'acquisition de richesses ne peut être une fin en soi. D'ailleurs l'Economique ne constitue pas une discipline autonome; elle appartient au champ de l'Ethique et de la Justice. Aristote souhaite créer l'harmonie dans la cité et pense que le bonheur peut être atteint sur terre à travers la recherche d'une cité idéale profitable à chacun. Le détachement par rapport aux richesses matérielles sous-tend sa volonté d'améliorer la vie sociale.

\footnotetext{
${ }^{5}$ Ethique à Nicomaque, I, 6, 1098 a.

${ }^{6}$ La politique, liv. I, chap. III, § 16.

${ }^{7}$ Ibid., § 23.

${ }^{8}$ La politique, liv. I, chap. III, § 23.
} 
Quelques années après la crise dite des subprimes et à l'heure où nos pays ne semblent parfois plus en mesure de rembourser les seuls intérêts de leur dette sans s'endetter davantage, l'acuité des conseils du philosophe grec mérite notre attention. Cette éthique aristotélicienne ne peut-elle être source de réflexion dans le management moderne ? Nous avons, certes, besoin de la finance, de la circulation de la monnaie, de la production de richesses, mais au service de l'Homme.

\section{B. Le bien commun et l'argent dans la philosophie de Saint Thomas d'Aquin}

Le concept de Bien commun figure dans la théologie chrétienne, à partir de Thomas d'Aquin, au XIIlème siècle, où il désigne l'inclinaison naturelle de la Création dans son ensemble (dont la communauté humaine) vers le Bien suprême qui est Dieu. "Thomas d'Aquin reçoit d'Aristote la structure de sa philosophie politique. Mais, en théologien, il l'insère dans un ordre chrétien de pensée du politique " (Daguet, 2014). Pour Thomas, le souverain bien, la fin recherchée ne peut être que Dieu à la fois cause première et finale de toute chose.

Comme le souligne Modde : "Il faut s'efforcer de revivre l'attitude théocentrique de l'esprit médiéval, selon laquelle tout l'univers animé et inanimé chante la louange du Créateur. Notre époque est tellement habituée à voir dans la communauté humaine ou dans l'Etat un tout autonome [...] que l'on ne songe plus à accorder grande importance aux réalités plus profondes qui peuvent régir l'humanité. Tout s'obscurcit alors. On ne voit plus la liaison nécessaire entre la vie individuelle et l'Etat, entre la prospérité de l'Etat et le bien de l'individu. Dans sa première conception du Bien commun, le Docteur Angélique dépasse cette vue superficielle ". Ainsi, pour Saint Thomas, le Bien commun doit être saisi à la fois au niveau surnaturel et au niveau naturel puisque, "Dieu est le bien commun transcendant, ou extrinsèque ", comme le précise Daguet (2014). La fin ultime de la vie dépasse cette terre. Cela ne disqualifie pas l'ordre naturel mais l'élève.

Saint Thomas sauvegarde la personne d'une emprise totale de la cité, en indiquant comme norme directrice la position de la cité : un tout relatif ; il dépasse de loin Aristote, pour qui la cité est le tout suprême. Chez Saint Thomas « le Bien commun de la cité est essentiellement moyen, puisqu'il est utilité commune ; il est en même temps fin, non pas fin ultime, mais fin intermédiaire. Il est moyen vis-à-vis du bien final de l'individu, du plein développement de sa personnalité morale dans l'épanouissement de sa vie intérieure de charité ; il est fin en tant que sa réalisation précède et conditionne l'acquisition du bien final ", selon Modde. "Toute la doctrine sociale de saint Thomas reste incompréhensible, si l'on ne tient pas compte de cette liaison étroite entre l'activité humaine et la béatitude. L'une est fin, l'autre est moyen ${ }^{9}$. Saint Thomas affirme alors dans la Somme théologique ${ }^{10}:$ "Celui qui cherche le bien général de la multitude cherche conséquemment son bien propre ". L'une des raisons est que "le bien propre ne peut exister sans le bien général de la famille, ou de la cité ou du royaume ${ }^{11}$. Toutefois il ne définit jamais vraiment clairement le Bien commun, même si la notion est très présente dans ses écrits.

\footnotetext{
${ }^{9}$ Modde.

$10 \|^{\mathrm{a}}-I^{\mathrm{ae}}$, qu. 47 , art. 10.

11 Ibid.
} 
Dans l'ordre financier, en quoi le Bien commun peut-il consister? Thomas ne sépare pas l'éthique et le politique. L'homme est un être d'action. Ses actes doivent être moralement justes et donc éthiques, guidés par l'amour du prochain et orientés vers Dieu. A partir de cette approche, il aborde les questions financières ${ }^{12}$ des achats et du prêt en s'appuyant sur les notions de justice, de partage et de charité dans la mesure où « le bien d'un seul homme n'est pas une fin dernière, mais il se rapporte au bien général ${ }^{13}$. Dans cette optique, Thomas d'Aquin ne suivra pas Aristote dans sa condamnation du commerce proprement dit. II admet que le profit modéré n'est pas nécessairement contraire à la vertu, si l'intention du commerçant est moralement bonne : soutenir sa famille, les indigents ou son pays. Dans la scolastique, le profit peut aussi être assimilé à une sorte de salaire qui récompense la peine, l'effort fourni ou le risque.

\section{Le bien commun et l'argent dans la doctrine sociale de l’Eglise}

Plus récemment, le Compendium de la doctrine sociale de l'Eglise reprend cette notion de bien commun ( $\S 164$ ): "Le bien commun ne consiste pas dans la simple somme des biens particuliers de chaque sujet du corps social. Etant à tous et à chacun, il est et demeure commun, car indivisible et parce qu'il n'est possible qu'ensemble de l'atteindre, de l'accroître et de le conserver, notamment en vue de l'avenir ". Toutefois, selon cette doctrine, "le bien commun de la société n'est pas une fin en soi ; il n'a de valeur qu'en référence à la poursuite des fins dernières de la personne et au bien commun universel de la création tout entière » ( $\S$ 170).

Après avoir précisé ( $(7)$ que " $c^{\prime}$ est une exigence de la justice et de la charité que de vouloir le bien commun et de le rechercher ", l'encyclique Caritas in veritate aborde plus spécifiquement les questions financières et les voies de développement. "L'économie et la finance, en tant qu'instruments, peuvent être mal utilisées quand celui qui les gère n'a comme point de référence que des intérêts égoïstes. Ainsi peut-on arriver à transformer des instruments bons en eux-mêmes en instruments nuisibles. Mais c'est la raison obscurcie de l'homme qui produit ces conséquences, non l'instrument lui-même. C'est pourquoi, ce n'est pas l'instrument qui doit être mis en cause mais l'homme, sa conscience morale et sa responsabilité personnelle et sociale "(§36). Comme voies de développement, l'encyclique suggère la gratuité, la solidarité qui ne peut " être déléguée seulement à l’Etat " (§ 38), le développement des " organisations productrices qui poursuivent des buts mutualistes et sociaux » (§ 38$)$, le crédit coopératif $(\S 65)$, le microcrédit $(\S 45)$ et la microfinance $(\S 65)$ qui rappelle le fonctionnement des Monts-de-Piété créés pour protéger « les couches les plus vulnérables de la population [...] contre les risques du prêt usuraire ou du désespoir ». L'encyclique met toutefois en garde (§ 45) sur l'existence d'une finance "éthique " à cause d'un étiquetage extérieur et non intrinsèquement. II s'agit donc de bien discerner "l'esprit ». Ces différentes pistes et les conséquences qui en découlent quant à l'utilisation de l'argent seront développées dans la partie III.

\footnotetext{
12\|\|$^{\mathrm{a}-\|^{\text {ae }}}$, qu. 77, “De la fraude que l'on commet dans les achats et les ventes et $\left\|^{\mathrm{a}}\right\|^{\mathrm{ae}}$, qu. 78, "Du péché de l'usure que l'on commet dans les prêts" ".

$\left.{ }^{13}\right|^{\mathrm{a}}-\|^{\text {ae }}$, qu. 90 , art. 3.
} 
Nous déplorons toutefois qu'aujourd'hui ce " bien commun " soit devenu assez flou pour se prêter aisément à tous les détournements politiques, voire à la restriction de nos libertés. Cette notion a envahi le débat public et déborde désormais de la conception classique. Aussi, ce trop rapide détour philosophique et théologique nous a paru nécessaire car il éclaire les soubassements intellectuels de la notion de Bien commun qui doit guider les décisions financières.

\section{Comment utiliser l'argent en vue du bien commun selon l'approche thomiste?}

Pour Saint Thomas, la cité est une organisation politique d'une communauté de personnes, finalisée par le Bien commun, informée par la justice et gouvernée selon la prudence. La question est alors de savoir quel rôle peut tenir la finance dans la poursuite de ce Bien commun. Saint Thomas ne permet sans doute pas de penser un ordre financier aujourd'hui mais il aide peut-être à comprendre les pathologies actuelles de la finance. Dans la Somme théologique il s'intéresse à la question " du péché de l'usure que l'on commet dans les prêts " $\left(\left\|^{a}-\right\|^{\text {ae }}\right.$, qu. 78) et à la question " de la fraude que l'on commet dans les achats et les ventes" (II ${ }^{\mathrm{a}}$-II ${ }^{\mathrm{ae}}$, qu. 77). II s'agit donc ici d'examiner les concepts sous-jacents dans sa doctrine pour ensuite définir des voies de développement de la finance vers le Bien commun.

\section{A. Le prêt d'argent : principes thomistes}

Il semble tout d'abord primordial de distinguer, comme en Chaldée il y a 4000 ans, d'une part, le prêt à la production et, d'autre part, le prêt à la consommation. Les théologiens se fondent eux sur la distinction romaine entre les biens non fongibles (ou durables) et les biens fongibles (ou consommables).

L'argent s'apparente au vin qui est un bien fongible : on ne peut pas dissocier son usage de sa propriété et vendre les deux séparément à différentes personnes contrairement à un bien immobilier, par exemple. "Si quelqu'un voulait vendre le vin d'une part et qu'il voulût d'une autre part en céder l'usage, il vendrait la même chose deux fois, ou il vendrait ce qui n'existe pas : il ferait donc évidemment une injustice ", commente Thomas d'Aquin ${ }^{14}$. De même, la monnaie existe pour être consommée dans l'échange quelle que soit la finalité. II conclut alors: " puisqu'on ne fait usage de l'argent qu'en le consommant et en le dissipant, il est injuste et illicite de recevoir quelque chose pour son usage ".

Pour les biens fongibles, le seul contrat acceptable est donc le mutuum ou prêt gratuit par lequel la propriété du bien est transférée à l'utilisateur qui a l'obligation de rendre, ensuite, le même type et la même quantité de biens. Le contrat, emprunté au droit romain, s'apparente à une vente et non à une location. On ne peut donc pas exiger « deux compensations » : la

$14\left\|^{\mathrm{a}}-\right\|^{\mathrm{ae}}, \mathrm{qu} .78$, art. 1. 
restitution d'une même quantité d'argent et le prix de son usage, ou "l'usure ". Les choses qui se consomment par l'usage ne sont pas susceptibles d'usufruit.

En revanche, pour les biens non fongibles, comme une maison ou une terre, l'usage est séparable de la propriété. On peut alors, selon Thomas d'Aquin ${ }^{15}$, « licitement retirer quelque chose pour l'usufruit d'une maison et en exiger en plus de l'entretien, comme on le voit dans les baux et les locations ". Dans ce cas, deux modalités sont alors parfaitement légitimes ; on peut envisager des contrats de prêt gratuit, commodatum ${ }^{16}$, ou bien des contrats de prêt non gratuit, locatio. Dans le cas de la location, le propriétaire cède l'usage du bien, mais en garde la nue-propriété et le locataire acquitte le prix de l'usage de ce bien non fongible.

La position de Thomas d'Aquin ${ }^{17}$ est très claire : « il n'est pas permis de recevoir en retour du prêt quelque chose d'estimable à prix d'argent, à moins qu'on ne le reçoive à titre de don gratuit », affirme-t-il en s'appuyant sur Ezéchiel (XVIII, 17) « il n'a pris ni usure, ni intérêt ». En revanche, il admet que " celui qui prête peut convenir avec l'emprunteur d'une indemnité pour la perte que lui cause la privation de l'argent qu'il devrait avoir. Car ce n'est pas là vendre de l'argent, $c^{\prime}$ est éviter une perte ${ }^{18}$. Cette disposition, le lucrum cessans (gain manqué), est utilisée pour permettre au prêteur d'obtenir le même profit qu'il aurait pu avoir dans un emploi différent de son argent. Le théologien introduit donc la notion de rémunération du manque à gagner ou de coût d'opportunité. II prévoit également d'autres «titres extrinsèques " qui permettent au créancier la perception éventuelle, en toute justice, d'un surplus au-delà du remboursement de la somme prêtée afin de compenser les dommages subis par le créditeur à cause de l'emprunteur, précise McCall (2008, page 570). Il peut s'agir, par exemple, d'un défaut de remboursement à la date prévue (damnum emergens ou poena) ou d'une perte liée à l'immobilisation de la somme prêtée. Pour les moralistes chrétiens, c'est l'esprit de la loi qui prévaut.

Rémunérer la contrepartie d'un coût d'opportunité est donc licite mais s'approprier le surplus d'un échange grâce à son pouvoir de négociation est prohibé par les théologiens. Il s'agit bien d'analyser l'intention pour déterminer le sens moral ou non du prêt.

L'autre apport de Saint Thomas concerne le partage du gain, du croît, selon une clef définie ex ante. En fonction de l'objet des prêts, Thomas d'Aquin ${ }^{19}$ définit des règles différentes : " celui qui prête de l'argent transfère la propriété de son argent à l'emprunteur ; par conséquent celui qui emprunte possède la somme à ses risques et périls et il est tenu de la rendre intégralement. Le prêteur ne doit donc pas exiger davantage. Mais celui qui prête son argent à un marchand ou à un artisan avec lequel il s'est associé ne lui transmet pas la propriété de la somme, il en reste toujours le propriétaire, de telle sorte que c'est à ses risques et périls que le marchand commerce sur son argent ou que l'artisan travaille. C'est pourquoi il peut

\footnotetext{
$15\left\|^{\mathrm{a}}-\right\|^{\mathrm{ae}}$, qu.78, art. 1.

${ }^{16}$ Le commodatum ou commodat est un contrat de prêt gratuit pour un bien non-consommable avec l'obligation de rendre ensuite le même bien.

$17\left\|^{\mathrm{a}}-\right\|^{\mathrm{ae}}, \mathrm{qu} .78$, art. 1.

18 Ibid.

${ }^{19}\left\|^{\mathrm{a}}-\right\|^{\mathrm{ae}}$, qu. 78 , art. 2.
} 
licitement recevoir une partie du gain qui résulte de là, comme étant le fruit de la chose ". Ici, l'argent est productif et le prêteur court un risque; il ne s'enrichit pas d'une façon disproportionnée eu égard à la responsabilité engagée. Dans cette hypothèse, ce n'est plus un simple prêt, mais un contrat de société. Des pertes potentielles du prêteur doivent, par symétrie, résulter des gains potentiels, ce qui s'apparente aux actions et aux dividendes actuels. Ainsi, la présence d'un risque concernant l'issue des opérations " justifiera, aux yeux du moraliste, la revendication d'un revenu spécifique lorsque ce risque est associé à la propriété. Bien sûr, cette association est fragile et les menaces qui pèsent sur elle laissent entrevoir le jour où propriété et risque feront l'objet de transactions séparées " (Lapidus, 1991).

II ne faut donc pas réduire le prêt à intérêt à son côté matériel : la livraison à l'emprunteur d'une certaine quantité de monnaie. Il s'agit bien de prendre en compte son côté économique : ce capital donne à l'emprunteur la faculté de se procurer des instruments de production et par suite d'accroître le rendement de son patrimoine. L'intérêt n'est autre chose qu'une part de ce croît lorsque le prêt sert à investir. Il est donc juste de partager le bénéfice qui résulte du prêt.

L'existence d'intérêt dans les prêts est très fluctuante selon les époques et les religions. Dans I'Antiquité, en Grèce, le prêt à intérêt n'était pas prohibé. A Rome, diverses lois se succédèrent pour limiter le taux d'intérêt en édictant des peines, sans succès. Elles furent contournées par des supercheries. En France, la loi religieuse interdisait l'intérêt ainsi que la loi civile, jusqu'au décret de l'Assemblée nationale du 3 octobre 1789. Après la Révolution française, l'activité bancaire et le prêt à intérêt devinrent libres. Toutefois, dès 1807, l'intérêt conventionnel fut limité par la loi du 3 septembre à $5 \%$ en matière civile et à $6 \%$ en matière de commerce pour éviter l'usure. Aujourd'hui, en France, la loi Lagarde de 2010 plafonne l'intérêt par un taux défini chaque trimestre par la Banque de France en fonction des taux effectifs moyens, pratiqués par les établissements de crédit pour chaque catégorie de prêt. L'objectif est d'éviter les abus et le surendettement des ménages. Ainsi, le processus continu de modification de la loi a souvent cherché à fixer les limites de l'intérêt pour protéger l'emprunteur.

\section{B. La spéculation : principes thomistes}

Dans son étude des achats et des ventes, Saint Thomas s'appuie sur les notions de finalité de l'acte, de juste prix et d'utilité.

Le gain « n'implique rien non plus qui soit absolument vicieux ou contraire à la vertu. Par conséquent, rien n'empêche qu'on ne le destine à une fin honnête ou nécessaire, et alors le négoce est permis ", affirme le moraliste ${ }^{20}$ qui distingue donc deux fins opposées : la nécessité et le gain. On ne peut pas être plus clair et plus nuancé. En toute chose, c'est la fin qui doit être recherchée. Notre auteur estime ainsi que le métier de négociant est dangereux car la recherche de l'argent, qui tend à devenir infinie, n'est pas, de soi, un but moral. Spicq (1934) s'insurge alors à propos de Thomas d'Aquin: "Il est impossible de lire ces textes sans

\footnotetext{
$20\left\|I^{\mathrm{a}}-\right\|^{\mathrm{ae}}$, qu. 77, art. 4.
} 
étonnement, voire sans scandale, mais ils s'inspirent des principes absolus de la finalité humaine, et non de la devise des boutiquiers romains, Salve lucrum ! ».

Toutefois Thomas va plus loin et accepte clairement le négoce. A la question ${ }^{21}$ «Est-il permis dans le commerce de vendre plus cher qu'on achète ? ", il répond que le négoce est permis " quand quelqu'un cherche à faire dans le commerce un bénéfice modéré pour soutenir sa famille ou pour secourir les pauvres; ou quand quelqu'un s'applique au commerce dans l'intérêt général, pour que sa patrie ne manque pas du nécessaire ". Il juge le commerce en théologien et en moraliste, non en économiste.

En effet, pour Thomas d'Aquin, la valeur d'un bien est avant tout morale. C'est la résultante d'une entente entre deux personnes dans le cadre d'un échange, elle est déterminée par rapport à autrui et correspond à un équilibre tâtonnant qui ne lèserait aucune des parties contractantes. Il distingue ici la loi humaine et la loi divine. La loi humaine permet au vendeur de majorer le prix de sa marchandise et à l'acheteur de l'acheter moins cher, pourvu qu'il n'y ait pas de fraude et qu'on ne dépasse pas certaines limites. La loi divine va plus loin : elle considère comme un acte illicite le fait de ne pas observer l'égalité de la justice dans l'achat et dans la vente. Chacun doit choisir non son intérêt personnel mais le bien commun.

Il a cerné toute la complexité de la question : si le vendeur ne peut pas se prévaloir de la nécessité de l'acheteur pour vendre une chose plus qu'elle ne vaut, il accepte toutefois un prix variant selon les circonstances, les lieux et ne se base pas sur la valeur intrinsèque du bien, déterminable a priori et de manière exogène au marché. "Le juste prix n’a pas été ponctuellement déterminé $"$, précise-t-il|22.

II ne construit pas non plus une approche fondée sur le prix concurrentiel, mettant en jeu le mécanisme de l'offre et de la demande qui prévaut aujourd'hui sur le marché financier. " $\mathrm{Ce}$ n'est qu'assez tardivement, sous l'influence, au XVlème siècle, de l'Ecole de Salamanque et de son fondateur F. de Vitoria, que les mécanismes de marché furent explicitement pris en considération » (Lapidus, 1986).

Mais les théologiens font remarquer que le prix des marchandises doit être proportionné à l'utilité que les hommes peuvent en retirer, ce qui fait référence à la valeur d'usage. Le prix juste de la chose va dépendre du jeu subtil des avantages et préjudices que la vente et l'achat peuvent induire chez l'un ou l'autre acteur.

Thomas distingue la justice commutative et l'amitié utile. Dans la justice commutative, on considère principalement l'égalité des choses échangées (capacité des produits à entrer en rapport quantifiable entre eux). Mais dans l'amitié utile, on considère l'avantage qu'on en a retiré. C'est pourquoi la récompense doit être alors proportionnée à l'utilité qu'on en retire, tandis que dans l'achat elle doit être égale à la chose.

La scolastique médiévale aide à concevoir des voies de développement pour la finance en accord avec la notion de Bien commun.

\footnotetext{
21 Ibid.

$22\left\|^{\mathrm{a}}-\right\|^{\mathrm{ae}}$, qu.77, art. 1.
} 


\section{Quelles pistes pour répondre aux défis à venir ?}

On observe une remise en question du système financier actuel dans le monde académique et dans la population en général. Les Etats sont de plus en plus confrontés, pour contrôler les acteurs, à l'opacité croissante des marchés et à la mondialisation. Les phénomènes de mimétisme conduisent les agents dans la même direction et génèrent des bulles spéculatives, causes de déséquilibres sur le prix des biens, de risques systémiques et d'emballements nocifs pour la collectivité (crise grecque, subprimes). Ils sont aujourd'hui amplifiés par l'évolution technologique avec le trading algorithmique qui optimise les tâches de prise de décision d'investissement et le trading à haute fréquence qui multiple le nombre d'ordres boursiers inaccessibles aux analyses humaines et exécutés en quelques microsecondes par informatique donc par des opérateurs virtuels.

Cette déconnection croissante entre le besoin effectif de marchandises, l'économie réelle et les marchés financiers nuit à la formation des prix et peut être la cause d'insécurité, de dynamiques incontrôlables, voire de famine. Ainsi, en 2007, la multiplication par deux du prix du contrat à terme sur le blé coté sur Euronext NYSE Life causa des émeutes de la faim dans quarante pays d'Afrique et d'Asie.

Le Rapport d'enquête sur les mécanismes de spéculation affectant le fonctionnement des économies, présenté à l'Assemblée Nationale, regrette " un déficit de gouvernance mondiale face à l'ampleur des enjeux " dans un contexte où " le déficit de coordination des politiques nationales ne permet pas d'éviter une instabilité qui se diffuse à l'ensemble de la planète ». L'étude propose alors de développer " une réglementation plus contraignante » des marchés, d'augmenter les " moyens des autorités de régulation et de supervision ", d'encadrer rigoureusement les banques et de favoriser "une coordination transatlantique ". Ainsi, l'intervention n'est plus possible seulement au niveau de l'Etat, présenté par Saint Thomas comme un instrument au service du Bien commun, dans la mesure où la finance est devenue supranationale dans un monde globalisé ; c'est d'ailleurs ce qui rend ses dérives actuelles difficilement contrôlables.

Dans ce contexte, quelles sont les pistes de développement apportées par la notion de Bien commun dans sa dimension prospective et par la théorie thomiste pour répondre au défi de demain de la vocation sociale de l'activité économique et financière ? Notre réflexion s'appuie sur les idées de partage équitable du gain, d'entraide par le don et de valeur d'usage mises en exergue par le Docteur Angélique.

\section{A. L'émergence des modalités de financement par le partage}

A Babylone (1793-1750 avant J.-C.) la notion d'intérêt était désignée par le mot sibtu, qui signifie le croît, l'accroissement d'un capital. En ce qui concerne les céréales, si le prêt était gratuit, le prêteur faisait donation de tout le croît. Dans le cas contraire, il pouvait, tout en rendant service à l'emprunteur, lui demander de partager avec lui une proportion déterminée du croît du capital. Dans le prêt à la production, nous retrouvons chez Saint Thomas et dans la 
finance islamique cette notion de partage, qu'il serait souhaitable de favoriser. Dans la mesure où il y a partage des risques, les bénéfices peuvent être partagés ex post entre les parties prenantes selon une clé définie ex ante. Dans la finance islamique les contrats moudharaba ou mousharaka s'appuient sur ce principe.

On retrouve aujourd'hui ce concept de partage de la ressource dans le financement participatif ou crowdfunding qui se développe et permet de financer l'économie réelle mais dans lequel la clé de répartition des bénéfices n'est pas définie ex ante. Dans ce système de financement désintermédié, de nombreuses personnes financent un projet qu'elles ont choisi de soutenir, souvent dans les TPE/PME et sans l'aide des acteurs traditionnels (les institutions financières ou les établissements de crédit). Les plateformes de financement se développent grâce à internet ou aux réseaux sociaux et $7 \%$ des français ${ }^{23}$ ont déjà donné, prêté ou investi grâce à ce système. Cette tendance s'inscrit dans un mouvement plus global de la consommation collaborative et de la production participative. L'investisseur a une visibilité sur la destination et l'utilisation de son argent et donne du sens à son investissement.

Le partage des finances est aussi l'objet des CIGALES (Clubs d'Investisseurs pour une Gestion Alternative et Locale de l'Epargne Solidaire). Ces groupes de 5 à 20 personnes épargnent mensuellement pour s'investir solidairement dans des petits projets d'entreprise, souvent locales, et mettent de surcroît leurs compétences à la disposition des sociétés soutenues.

Le partage de l'argent est promu également par la finance solidaire dont le développement est récent sous ses formes institutionnelles actuelles, même si les valeurs dont elle est porteuse sont plus anciennes. Actuellement, les finances solidaires regroupent un ensemble d'organismes qui fournissent des prêts ou des participations en capital à d'autres structures de l'économie sociale et solidaire (associations, coopératives), à des personnes exclues des circuits bancaires classiques, à des entreprises favorisant l'agriculture biologique ou créées par des personnes en difficulté, par exemple. Elles reçoivent l'épargne d'investisseurs souhaitant que leurs fonds, vus comme "commun", soient placés dans une démarche d'entraide en acceptant d'en retirer une rémunération parfois un peu moins élevée.

Si toutes les banques françaises proposent des placements éthiques ${ }^{24}$, fondés sur une logique d'exclusion, elles ne proposent pas toutes, en revanche, des placements solidaires, basés sur une approche d'inclusion. Ces fonds sont développés notamment par le Crédit coopératif. Les autres banques coopératives, comme le Crédit agricole et le Crédit mutuel, par exemple, les commercialisent également, sans que cela ne représente toutefois un pourcentage vraiment significatif de leur activité. Ainsi, fin 2016, l'épargne financière des Français s'établissait à 4764,7 milliards d'euros, selon la Banque de France, et la part de l'épargne solidaire dans ce patrimoine financier ne s'élevait qu'à $0,20 \%$ (doublement ${ }^{25}$ en 5 ans).

Nous pouvons enfin citer le développement récent des social bonds ou obligations ISR (Investissement Socialement Responsable). Créées avec un objectif d'entraide, ces obligations financent des projets ayant un impact social sur la vie des communautés en facilitant, par

\footnotetext{
${ }^{23}$ Sondage réalisé par l'Institut Think pour Lendopolis et le Conseil Supérieur de I'Ordre des Experts-Comptables à l'occasion du $22^{\text {ème }}$ Salon des Entrepreneurs, édition 2015.

${ }^{24}$ Les fonds éthiques excluent des secteurs considérés «non éthiques » : les armes controversées (mines antipersonnel, armes biologiques, chimiques), le tabac ou, dans la finance islamique, le porc, la pornographie ou l'alcool.

${ }^{25}$ Rapport Finansol, Zoom sur la finance solidaire, 2017.
} 
exemple, l'accès à l'école ou la création d'emploi. En 2012, Air Liquide fut le premier émetteur privé à se lancer sur ce marché pour financer l'activité de soins à domicile du groupe avec un coupon à taux fixe de 2,125\%. En 2018, Danone a émis un social bond affecté à la recherche sur la nutrition et à l'aide au développement d'une agriculture responsable. Le succès auprès des investisseurs a permis à l'émetteur de réduire le rendement offert qui ressort à 1,02\% pour une maturité de 7 ans.

Les notions de finalité de l'acte et de partage de la ressource, mises en évidence par Saint Thomas, sont bien présentes dans ces produits financiers, certes marginaux. Les produits dits de " partage " proposent même d'aller plus loin avec le don d'une partie du gain du titulaire du compte. Dans cette catégorie de fonds solidaires, une fraction des intérêts est en effet reversée aux associations sous forme de don et, en contrepartie, l'épargnant bénéficie d'un cadre fiscal avantageux.

\section{B. La nécessité de concevoir un prêt " gratuit »}

Pour justifier la nécessité du prêt gratuit, les théologiens du Moyen-Âge s'appuient sur des textes religieux. L'Ancien Testament ${ }^{26}$ est sans équivoque sur l'interdiction du prêt à intérêt dans l'Exode (XXII, 24) : "Si tu prêtes de l'argent à quelqu'un de mon peuple, au pauvre qui est avec toi, tu ne seras point à son égard comme un créancier, tu n'exigeras pas de lui d'intérêt ". Le Coran condamne également le prêt à intérêt sans ambigüité : "Ô croyants ! Craignez Dieu ; et renoncez au reliquat de l'intérêt usuraire, si vous êtes croyants. Et si vous ne le faites pas, alors recevez l'annonce d'une guerre de la part de Dieu et de Son messager » (Sourate II, versets 278 et 279). L'idée est de développer l'entraide. Aussi observe-t-on dans I'histoire que les prêts à la consommation gratuits soudent les membres de la communauté et contribuent à son existence, comme le prouve le recueil des lois promulguées à Babylone par le roi Hammourabi.

Dans cet esprit, le microcrédit a été créé en 1976 par un économiste du Bangladesh pour octroyer de petits prêts presque "gratuits", de faible ampleur et de courte durée aux personnes exclues du système bancaire traditionnel pour leur permettre d'acheter des matières premières ou des poules afin de développer une activité économique. Le pari était de favoriser un écosystème qui permette à des familles, des tribus, des villages de trouver un équilibre économique et de subvenir à leurs besoins primaires. Le microcrédit a trouvé son essor dans les pays en voie de développement car il y avait un besoin réel de financement de création d'entreprises et de développement d'infrastructures (puits, écoles, etc.) demandant très peu de capitaux.

Malheureusement, il a souvent échoué dans son rôle d'outil de lutte contre la pauvreté car les fonds étaient utilisés pour la consommation ou à cause de débouchés insuffisants pour l'activité ou encore quand le coût financier du prêt était supérieur au bénéfice monétaire de l'activité additionnelle créée. De surcroît, les projets financés par un partage de la ressource 26 D'autres textes réprouvent I'usure dans I'ancien Testament dans le Deutéronome (XXIII, 20-21) et dans le
nouveau Testament dans l'évangile de saint Luc (VI, 34). 
ont rarement été gérés comme des biens communs. Ainsi, considérer la source du financement comme un commun nécessite de porter une attention nouvelle sur le fonctionnement des structures et du secteur dans son ensemble. "Monnaie et finance peuvent devenir des communs, mais le renouvellement d'une ressource dans l'ordre monétaire et financier est différent de celui que l'on observe dans la nature. La condition première du renouvellement des prêts est leur remboursement: les prêts remboursés peuvent être prêtés à nouveau, soit à d'autres, soit aux mêmes emprunteurs dans une série successive de prêts", observe Servet. "On voit ainsi qu'en matière monétaire et financière, la défense des communs ne passe pas par une politique conservatrice ou protectrice de la ressource en tant que telle, mais suppose son renouvellement. Ce renouvellement par une réinjection permanente dans les circuits de financement s'oppose à une stérilisation de la ressource au nom d'une préservation des droits de propriété privés ${ }^{27}$. Ainsi, lorsque les prêts ne sont pas remboursés, la monnaie ne peut plus être utilisée comme commun. Or, petit à petit, les ménages avec un microcrédit ont commencé à contracter des prêts pour rembourser leurs premiers emprunts avec un enchainement de dette et un surendettement. Le taux de remboursement des ménages s'est effondré et les institutions de microfinance ont été touchées par une crise de liquidité provoquant des conséquences économiques et sociales désastreuses. De surcroît, les marchés financiers ont dévoilé leur appétit pour la microfinance et les nouveaux entrants ont proposé des taux de plus en plus élevés, d'où l'échec partiel de ce système de prêt.

A l'heure où, en France, la moitié des ménages est endettée, il s'agit d'explorer des formes justes et équitables de prêts développées dans un esprit de soutien mutuel à l'instar des Montde-Piété, ancêtre du Crédit municipal de Paris. Créés en Italie au XVème siècle pour combattre l'usure et permettre l'accès au crédit à moindre coût, ces établissements proposaient des prêts sur gage "gratuits", autrement dit à faible taux d'intérêt pour couvrir les frais de fonctionnement.

Ainsi, mettre la finance au service du Bien commun pourrait correspondre à développer une finance inclusive ${ }^{28}$, c'est-à-dire, une finance qui aide à combattre l'exclusion en tenant compte de l'apport pour la vie sociale et pas uniquement de la rentabilité financière.

\section{L'émancipation de la valeur d'usage}

Pour Saint Thomas la valeur d'un bien ne doit pas dépendre de la nécessité de l'acheteur mais de l'utilité, de l'usage, du bienfait que l'on peut en retirer. Elle est donc subjective et dépend de l'utilisateur et des circonstances. Cependant, à mesure que se sont effacés les idéaux médiévaux, s'est imposée une problématique de justice commutative, dans laquelle prévaut la valeur d'échange (déterminée par le marché) qui n'a alors cessé d'occulter la valeur d'usage et l'idée d'un bien commun avec l'utilité que les produits procurent aux consommateurs. Toutefois, ce concept de valeur d'usage resurgit depuis peu dans l'orbe de tout un ensemble de questionnements théoriques sur le caractère durable du développement économique et

\footnotetext{
27 Servet.

${ }^{28}$ L'inclusion financière (ou finance inclusive) est l'offre de services financiers et bancaires de base à faible coût pour des consommateurs en difficulté et exclus des services traditionnels. L'inclusion financière est donc le pendant de « l'exclusion bancaire » qui est directement liée à l'exclusion sociale.
} 
sur les craintes de ne pouvoir léguer aux générations futures les biens naturels. En effet, « des choses qui ont la plus grande valeur en usage n'ont souvent que peu ou point de valeur en échange ", précise Adam Smith. " II n'y a rien de plus utile que l'eau mais elle ne peut presque rien acheter ; à peine y a-t-il moyen de rien avoir en échange ${ }^{29}$.

Mais la valeur de l'environnement peut venir aussi bien de nos besoins présents que d'une utilité potentielle dans le futur. A partir de cette réflexion, la finance peut jouer un rôle positif dans les grands enjeux écologiques avec les green bonds (obligations vertes), émises par une collectivité locale ou une entreprise pour financer les projets à bénéfice environnemental (investissement dans les transports, gestion des ressources rares comme l'eau), et les climate bonds (obligations climat). Ces dernières sont un sous-ensemble des green bonds. Elles sont émises surtout par les grands financeurs publics internationaux ou par les entreprises (EDF, Engie) pour financer les projets qui limitent les émissions de carbone. La France est le deuxième émetteur de green bonds, derrière la Chine et devant les Etats-Unis. Ce marché prometteur a connu une très forte croissance depuis 2013. Toutefois, les green bonds ne représentent encore qu'une toute petite fraction (à peine $0,15 \%$ ) de l'ensemble des encours obligataires dans le monde.

Mais disons-le tout de suite, « il existerait une synergie positive entre la performance sociétale et la performance financière avec un "cercle vertueux" " (Marie-Jeanne) dans la mesure où les mauvaises pratiques environnementales, sociales et de gestion finissent toujours par aboutir à une destruction de valeur pour les entreprises concernées, sous forme d'amendes (pour pollution par exemple), d'indemnités ou de détérioration de l'image de marque. Les fonds ISR environnementaux, construits sur le thème de l'eau ou des énergies renouvelables, proposés par les banques, sont ainsi souvent très performants sur le plan de la rentabilité, d'où leur attrait financier.

Par ailleurs, réintroduire la valeur d'usage semble primordial à une époque où le prix des matières premières agricoles est lié aux aléas de la spéculation, sans tenir compte de la nécessité de l'acheteur ou du vendeur. A titre d'illustration, le squeeze consiste à créer une situation réelle ou artificielle de rareté du sous-jacent grâce à d'importants volumes d'achats sur le marché à terme et sur le marché physique, ce qui entraîne une hausse des prix avec des bénéfices élevés à la revente pour les spéculateurs et des famines pour les acheteurs tributaires de la valeur de marché, c'est-à-dire du prix, valeur d'échange.

Comment trouver un équilibre pour que la valeur d'usage soit prise en compte ? "Pour que la valeur d'usage s'exprime, il faut que les personnes portant le projet collectif affirment celleci en termes de bien-être collectif et de moyens à mobiliser pour y parvenir, avec la mise en place d'une finance cohérente avec elle ", propose Paranque.

${ }^{29}$ Smith. 
Ainsi, la philosophie thomiste nous invite à remettre en question les présupposés du système financier moderne basé sur la maximisation de la rentabilité à court terme et à réintroduire les notions de Bien commun et de partage qui étaient de plus en plus occultées. Cette étude a ébauché quelques pistes pour montrer comment la finance peut être un instrument au service du Bien commun, dans le cadre de la finance participative, de la finance solidaire ou des social bonds, et comment l'argent peut être destiné à un usage commun pour permettre à des communautés de développer une activité économique et de subvenir à leurs besoins, grâce au microcrédit. Par ailleurs, la finance semble être un soutien précieux pour la préservation de notre environnement avec les green bonds et les climate bonds.

Jean Tirole, prix Nobel d'économie, constate dans son livre Economie du bien commun que " même dans le "monde libre", le pouvoir politique a perdu de son influence, au profit de la loi du marché et de nouveaux acteurs ", comme les autorités indépendantes de régulation, organes non soumis à la primauté du politique. Cette finance autonome et indépendante à l'égard du politique peut-elle avoir le sens du Bien commun politique d'une Cité ? La mutualisation ou la finance alternative, en permettant de s'écarter de cette finance vagabonde, peuvent y contribuer. Si l'Etat a parfois des difficultés à pallier les défaillances du marché, notamment pour des raisons de territorialité juridictionnelle face à une finance typiquement apatride, il peut toutefois favoriser les placements financiers qui concourent au Bien commun en incitant ses entreprises et ses citoyens à investir dans des instruments $d^{\prime}$ 'entraide. Son intervention peut s'appuyer sur la loi qui est, selon Saint Thomas ${ }^{30}$, « un ordre de la raison tendant au bien général, promulgué par celui qui a soin de la communauté ». En ce sens, le droit est aussi le moyen pour une société de concevoir une réponse constructive aux dérives de la financiarisation de l'économie et d'ordonner la cité vers le Bien commun.

\section{Bibliographie}

- AQUIN T. (d'), (1852), Somme théologique, Traduction de C.-J. Drioux, Ed. Belin.

- ARISTOTE (1848), La politique, Traduction de J. Barthélémy-Saint-Hilaire, Dumont.

- ARISTOTE, (2014), Ethique à Nicomaque, Traduction (1959) J. Tricot, Editions Les Echos du Maquis.

- BENOIT XVI, (2009), Lettre encyclique Caritas in Veritate, Editions Saint-Paul.

- Conseil Pontifical " Justice et Paix », (2005), Compendium de la doctrine sociale de l'Eglise, Bayard-Cerf-Fleurus-Mame.

- DAGUET F., (2014), "Le bien commun dans la théologie politique de saint Thomas d'Aquin ", Revue thomiste, $\mathrm{n}^{\circ} \mathrm{CXIV}$, pp. 95-128.

- DARDOT P., LAVAL C., (2014), Commun - Essai sur la révolution au XXlème siècle, Paris, La découverte.

- LAPIDUS A., (1986), Le détour de valeur, Economica.

\footnotetext{
$30 \mu^{\mathrm{a}}-I^{\mathrm{ae}}$, qu. 90 , art. 4
} 
- LAPIDUS A., (1991), " Le cœur et les mains : le rôle de l'information dans la doctrine médiévale de l'usure ", Economie et sociétés, n¹5, mai, pp. 31-60.

- MANCEL J.-F. (2010), Rapport d'enquête sur les mécanismes de spéculation affectant le fonctionnement des économies, Assemblée Nationale, Rapport n ${ }^{\circ} 3034,14$ décembre.

- MARIE-JEANNE C., (2004), Finance et éthique, la réconciliation ?, Revue d'Economie Financière, $\mathrm{n}^{\circ} 74, \mathrm{p} .319-332$.

- MCCALL B.M., (2008), "Unprofitable Lending: Modern Credit Regulation and the Lost Theory of Usury ", Cardozo Law Review, vol. 30-2, January, pp. 548-615.

- MODDE A., (1949), "Le Bien Commun dans la philosophie de saint Thomas ", Revue philosophique de Louvain, vol. 47, n¹4.

- OSTROM E., (2010), Gouvernance des biens communs - Pour une nouvelle approche des ressources naturelles, De Boeck.

- PARANQUE B., (2016), La finance comme commun : un idéal-type pour des émancipations, Revue de la régulation, 20, $2^{\mathrm{ème}}$ semestre, Automne.

- PLATON, (2016), La République, Traduction Georges Leroux, Flammarion.

- SAMUELSON P., (1954), The Pure Theory of Public Expenditure, The Review of Economics and Statistics, vol. 36, n4., nov., p. 387-389.

- SMITH A., (1776), Recherche sur la nature et les causes de la richesse des nations, Edition traduite en 1881 par Germain Garnier.

- SERVET J.-M., (2015), La finance et la monnaie comme un " commun », Etude pour l'Institut Veblen pour les réformes économiques, mai.

- SPICQ C., (1934), La moralité du commerce - Les leçons d'une crise au XIIlème siècle, La Vie Intellectuelle, 25 avril, pp. 460-482.

- TIROLE J., (2018), Economie du bien commun, PUF. 